\title{
Chest X-ray in new Coronavirus Disease 2019 (COVID-19) infection: findings and correlation with clinical outcome
}

\author{
Diletta Cozzi ${ }^{1}$ (D) Marco Albanesi ${ }^{1,2} \cdot$ Edoardo Cavigli $^{1}$ (]) $\cdot$ Chiara Moroni $^{1} \cdot$ Alessandra Bindi $^{1} \cdot$ Silvia Luvarà $^{1}$. \\ Silvia Lucarini ${ }^{1} \cdot$ Simone Busoni $^{3}$ (1) $\cdot$ Lorenzo Nicola Mazzoni $^{3,4}$ (1) $\cdot$ Vittorio Miele $^{1}$ (])
}

Received: 5 May 2020 / Accepted: 25 May 2020 / Published online: 9 June 2020

(c) Italian Society of Medical Radiology 2020

\begin{abstract}
Aim The purpose of this study is to describe the main chest radiological features (CXR) of COVID-19 and correlate them with clinical outcome.

Materials and methods This is a retrospective study involving patients with clinical-epidemiological suspect of COVID-19 infection, who performed CXRs at the emergency department (ED) of our University Hospital from March 1 to March 31, 2020. All patients performed RT-PCR nasopharyngeal and throat swab, CXR at the ED and clinical-epidemiological data. RT-PCR results were considered the reference standard. The final outcome was expressed as discharged or hospitalized patients into a medicine department or intensive care unit (ICU).

Results Patients that had a RT-PCR positive for COVID-19 infection were 234 in total: 153 males (65.4\%) and 81 females (34.6\%), with a mean age of 66.04 years (range 18-97 years). Thirteen CXRs were negative for radiological thoracic involvement (5.6\%). The following alterations were more commonly observed: 135 patients with lung consolidations (57.7\%), 147 $(62.8 \%)$ with GGO, 55 (23.5\%) with nodules and 156 (66.6\%) with reticular-nodular opacities. Patients with consolidations and GGO coexistent in the same radiography were $35.5 \%$ of total. Peripheral (57.7\%) and lower zone distribution (58.5\%) were the most common predominance. Moreover, bilateral involvement $(69.2 \%)$ was most frequent than unilateral one. Baseline CXR sensitivity in our experience is about $67.1 \%$. The most affected patients were especially males in the age group 60-79 years old (45.95\%, of which $71.57 \%$ males). RALE score was slightly higher in male than in female patients. ANOVA with Games-Howell post hoc showed significant differences of RALE scores for group 1 vs $3(p<0.001)$ and 2 vs $3(p=0.001)$. Inter-reader agreement in assigning RALE score was very good (ICC: $0.92-$ with $95 \%$ confidence interval $0.88-0.95)$.

Conclusion In COVID-19, CXR shows patchy or diffuse reticular-nodular opacities and consolidation, with basal, peripheral and bilateral predominance. In our experience, baseline CXR had a sensitivity of $68.1 \%$. The RALE score can be used in the emergency setting as a quantitative method of the extent of SARS-CoV-2 pneumonia, correlating with an increased risk of ICU admission.
\end{abstract}

Keywords Infection · Coronavirus · COVID-19 $\cdot$ Chest radiography $\cdot$ Diagnostic imaging

Diletta Cozzi

dilettacozzi@gmail.com

1 Department of Emergency Radiology, University Hospital Careggi, Largo Brambilla 3, 50134 Florence, Italy

2 Department of Clinical and Experimental Medicine, Institute of Diagnostic Imaging 2, University of Sassari, Sassari, Italy

3 Medical Physics Department, University Hospital Careggi, Florence, Italy

4 Medical Physics Unit, AUSL Toscana Centro, Pistoia, Prato, Italy

\section{Introduction}

At the end of 2019 a novel virus, named SARS-CoV-2 (Severe Acute Respiratory Syndrome Coronavirus 2), expanded globally from China with the first Italian cases dating back to February 2020 [1]. This new coronavirus causes a highly infectious disease, commonly called Coronavirus Disease 19 (COVID-19): Lung infection can result in severe pneumonia up to more aggressive acute respiratory distress syndrome (ARDS) [2, 3]. Genetic sequencing of SARS-CoV-2 has permitted the rapid development 
of real-time reverse transcription polymerase chain reaction (RT-PCR) of viral nucleic acid, and nowadays this is the diagnostic gold standard [2]. However, this serologic examination has several limitations due to the high number of false-negative tests and the delayed results. Radiological evaluation of patients with clinical-epidemiological suspect of COVID-19 is mandatory, especially in the emergency department (ED) while waiting for RT-PCR results, in order to have a rapid evaluation of thoracic involvement. The recent COVID-19 radiological literature focuses primarily on computed tomography (CT) findings, which is more sensitive and specific than chest X-ray (CXR): In particular, in China CT is used as a first-line diagnostic method for COVID-19 [4, 5]. Nonetheless, it has to be remembered that performing CT scan is not easy during this pandemic, considering not only the excessive radiation exposure especially to younger patients but also the mandatory scanner disinfection procedures that have to take place. The most Italian hospitals are employing CXR as the first-line method, with faster results comparing with those of RT-PCR, especially by using portable $\mathrm{X}$-ray units which reduce the movement of patients and so minimizing the risk of cross-infection [6-8] . Therefore, the purpose of our study is to better understand the main radiographic features of COVID-19 pneumonia, by describing the main CXR findings in a selected cohort of patients, also correlating the radiological appearance with RT-PCR examination and patients outcome (intended as discharged or hospitalized into a medicine department or intensive care unit).

\section{Materials and methods}

\section{Patients selection and inclusion criteria}

CXRs of patients with clinical-epidemiological suspect of COVID-19 infection performed at the ED of our University Hospital from March 1 to March 31, 2020, were retrospectively reviewed. Inclusion criteria were: patients' age between 18 and 99 years, RT-PCR nasopharyngeal-throat swab and CXR performed immediately at the ED access, clinical-epidemiological data suspect for COVID-19 infection and their duration at the time of ED access (fever, cough, dyspnea, respiratory impairment, diarrhea, asthenia, myalgia and dysgeusia). RT-PCR results were considered the reference standard. For the radiological assessment we selected only patients with RT-PCR-positive results. The final outcome was expressed as discharged or hospitalized patients into a medicine department or into an intensive care unit (ICU).

\section{Image acquisition and analysis}

All CXRs were acquired as digital radiographs with the same portable X-ray unit (FDR Go PLUS-Fujifilm, Italia) in the isolation wards of our ED. CXRs were performed in the postero-anterior or antero-posterior projection. All images were stored in a picture archiving and communication system (PACS, Syngo-Siemens). An independent and retrospective review of each CXR was performed by two thoracic radiologists in order to define the number of radiological suspects of COVID-19 infection; after this, they defined the predominant pattern of COVID-19 pneumonia presentation in patients with a positive RTPCR. In case of discordance, a consensual agreement was reached. Radiographic features including consolidation, ground-glass opacities (GGO), pulmonary nodules and reticular-nodular opacities were diagnosed according to the Fleischer Society glossary of terms [9]. Moreover, CXRs were assessed for the presence of a specific distribution of the disease (mostly peripheral or perihilar predominance), monolateral (right or left lung) or bilateral disease, upper or lower or diffuse predominance. All thoracic images were also assessed for evidence of other associated pulmonary pathology (cardiomegaly, hilar vascular congestion, pleural effusion, pneumothorax). Finally, to quantify the extent of COVID-19 lung involvement, a severity score was applied (Radiographic Assessment of Lung Edema-RALE) [10]. Following RALE indications, each CXR was given a score between 0 and 48 , ranging from the absence of any pathological sign (score 0 ) to the complete pathological involvement of lung parenchyma (score 48). The score was separately assessed by each of the two radiographers.

\section{Statistical analysis}

Statistical analysis was performed with SPSS (SPSS Chicago IL, USA). Descriptive statistics of RALE score were calculated for each group of patients. Analysis of Variance (ANOVA) was performed to detect possible differences among RALE score estimated in the following groups: discharged patients, hospitalized patients into a medicine department, hospitalized patients into an ICU (respectively, group 1, 2 and 3 in the following). Homogeneity of the variance was established between groups by means of Levene test, to adequately choose the post hoc test: Bonferroni in case of detected significant homogeneity of variance, otherwise Games-Howell. Intraclass correlation coefficient (ICC) was calculated to assess inter-reader agreement in assigning RALE score. Statistical significance threshold was set at $p=0.05$. 


\section{Results}

We found 482 patients fulfilling the following selecting criteria: presence of clinical-epidemiological suspect of COVID-19 infection and RT-PCR and CXR performed at

Table 1 Radiographic findings of our cohorts of COVID-19 patients

\begin{tabular}{ll}
\hline COVID-19 Radiological features & $\mathrm{N}(\%)$ \\
\hline Normal baseline CXRs & $13(5.6)$ \\
Abnormal baseline CXRs & $223(94.4)$ \\
Reticular-nodular opacities & $156(66.6)$ \\
Ground glass opacities & $147(62.8)$ \\
Consolidation & $135(57.7)$ \\
Vascular congestion signs & $92(39.3)$ \\
Cardiomegaly & $70(29.9)$ \\
Nodules & $55(23.5)$ \\
Pleural effusion & $39(16.7)$ \\
Pneumothorax & $5(2.4)$ \\
Distribution: & \\
Peripheral & $135(57.7)$ \\
Perihilar & $51(20.7)$ \\
Diffuse & $99(41)$ \\
Basal predominance & $137(58.5)$ \\
Superior predominance & $31(13.1)$ \\
Right lung & $29(58)$ \\
Left lung & $21(42)$ \\
Bilateral & $162(69.2)$ \\
\hline
\end{tabular}

the ED admission. Patients with a RT-PCR-positive results for COVID-19 infection were 234: Of these, 153 were males $(65.4 \%)$ and 81 females (34.6\%), with a mean age of 66.04 years (range 18-97 years). Only 13 CXRs were negative for radiological thoracic involvement (5.6\%). The others showed variable features as described in Tables 1 and 2. The following alterations were more commonly observed: 135 patients with lung consolidations (57.7\%), 147 (62.8\%) with GGO, 55 (23.5\%) with nodules and $156(66.6 \%)$ with reticular-nodular opacities. Patients with consolidations and GGO coexistent in the same radiography were $35.5 \%$ of total. In RT-PCR-positive patients, we found also signs nonspecific for COVID-19 pneumonia as hilar or vascular congestion (39.3\%), cardiomegaly (29.9\%), pleural effusion (16.6\%) and pneumothorax (2.4\%). Peripheral (57.7\%) and lower zone distribution $(58.5 \%)$ were the most common predominance. Bilateral involvement (69.2\%) was most frequent than unilateral one. Given the results, baseline CXR sensitivity in our experience is about $68.1 \%$.

In our population the most affected patients were in the age group of $60-79$ years old $(43.6 \%$, of which $71.57 \%$ males); patients older than 80 years $(23.1 \%)$ often presented more advanced lung involvement (Fig. 1). Nine patients (4\%) were immediately discharged from ED, and the others were hospitalized in medicine department or ICU (Table 3). A total of $34(15.3 \%)$ patients died in the 30 days included in this study (9 in group 2 and 25 in group 3 ).

Descriptive statistics of RALE score for each group is reported in Table 4. RALE score was slightly higher in male than in female patients. Levene test showed significant

Table 2 Graphic of CXRs findings of our cohorts of COVID-19 patients

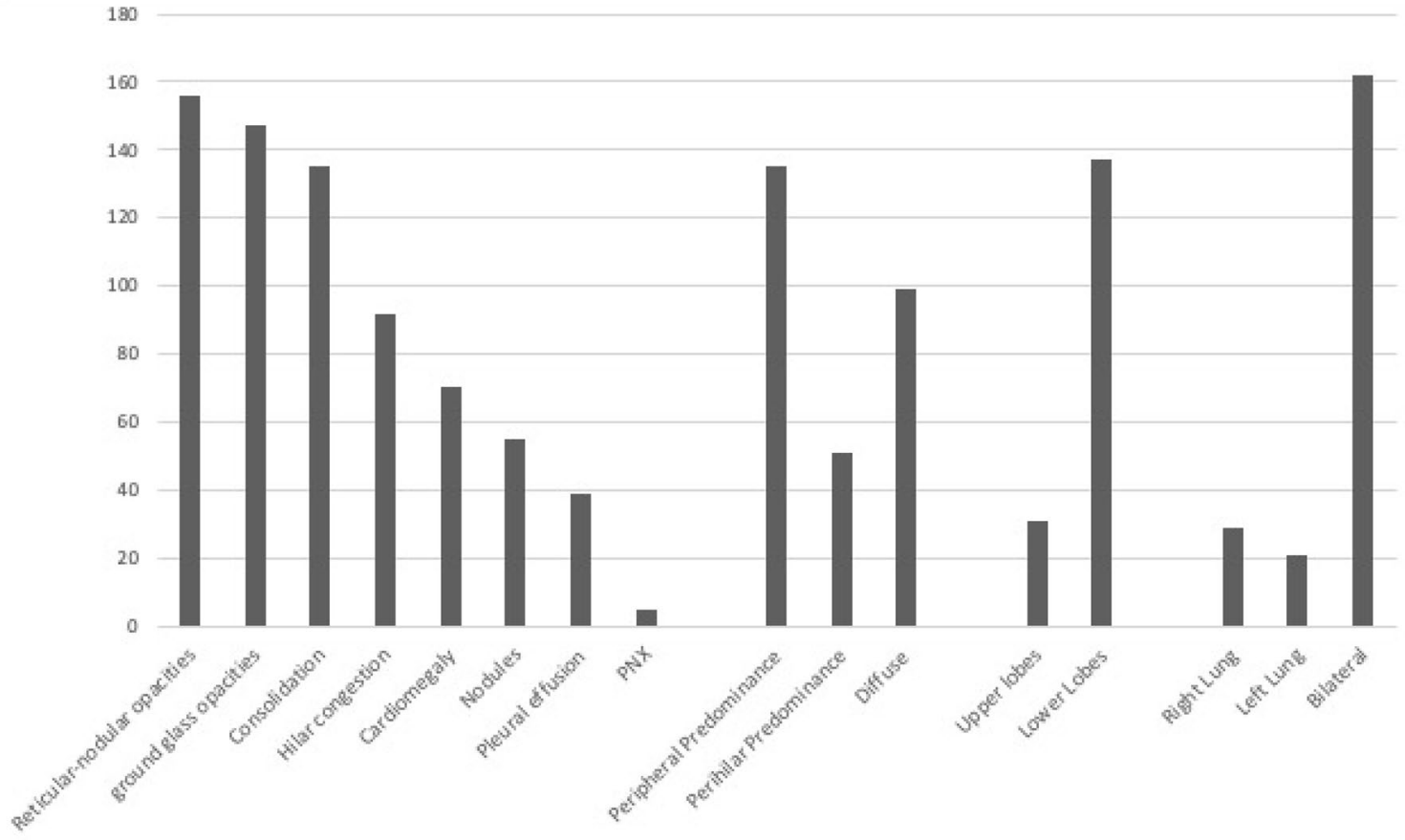




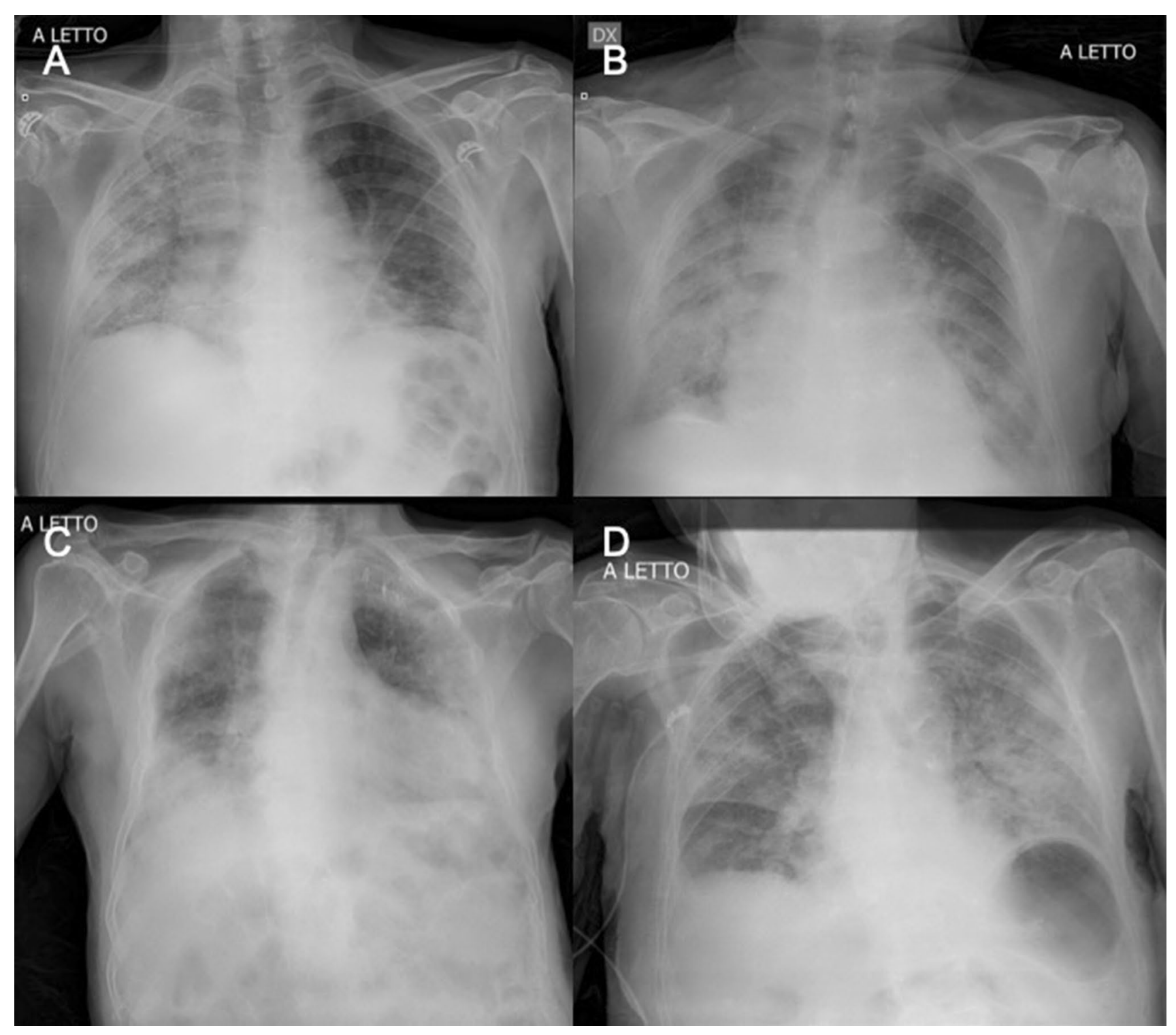

Fig. 1 Diffuse lung involvement in elderly patients. Four cases of advanced lung disease with diffuse consolidations and interstitial involvement of patients older than 80 years at the emergency department

inhomogeneities of variances among groups 1,2 and 3; thus, Games-Howell post hoc test was adopted. ANOVA with Games-Howell post hoc showed significant differences of RALE scores for group 1 vs group $3(p<0.001)$ and group 2 vs group $3(p=0.001)$. Box and Whisker plot of RALE score estimated in groups 1-3 is reported in Fig. 2. Inter-reader agreement in assigning RALE score was very good: ICC, with 95\% confidence interval in parentheses, was $0.92(0.88-0.95)$.

\section{Discussion}

In the context of a global pandemic, the radiological approach should be aimed at a rapid classification of the patient with suspected COVID-19 infection. All the Italian radiology departments are at the forefront in the diagnosis, in the quantification and in the follow-up of COVID-19 
Table 3 Age distribution of patients with positive swab for SARS-CoV-2 virus

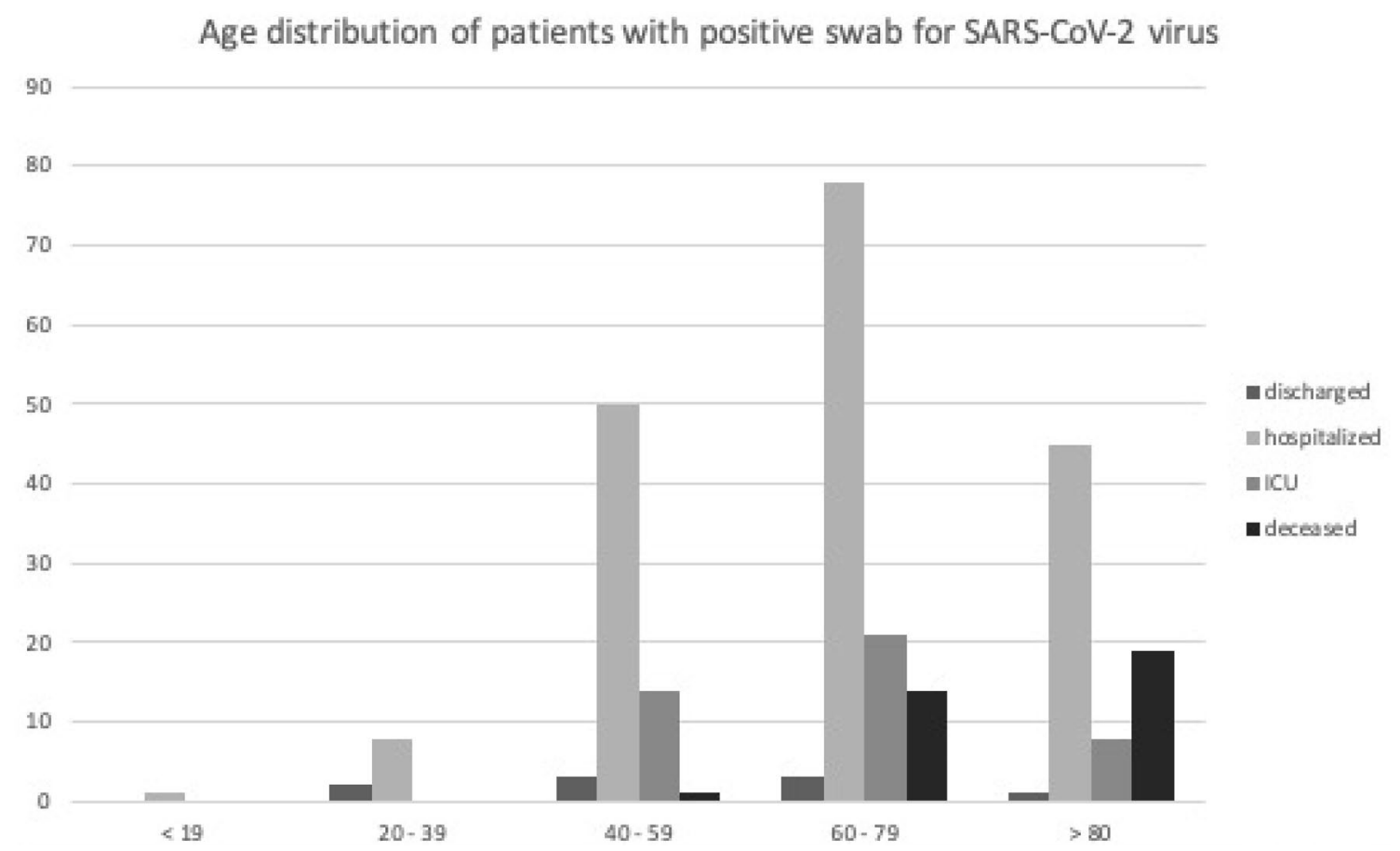

ICU intensive care unit

Table 4 RALE score in different groups of patients

\begin{tabular}{llllll}
\hline & Male $(N=153)$ & Female $(N=81)$ & Group 1 $(N=9)$ & Group 2 $(N=182)$ & Group 3 $(N=43)$ \\
\hline RALE score (mean; median; range) & $12.7 ; 10 ; 0-44$ & $10.2 ; 7.5 ; 0-42$ & $5.4 ; 4 ; 0-16$ & $10.6 ; 8 ; 0-40$ & $19.1 ; 16 ; 1-44$ \\
\hline
\end{tabular}

Mean, median and range of RALE score calculated for the following groups: male, female, discharged patients (group 1), hospitalized patients into a medicine department (group 2), hospitalized patients into an intensive care unit (group 3)

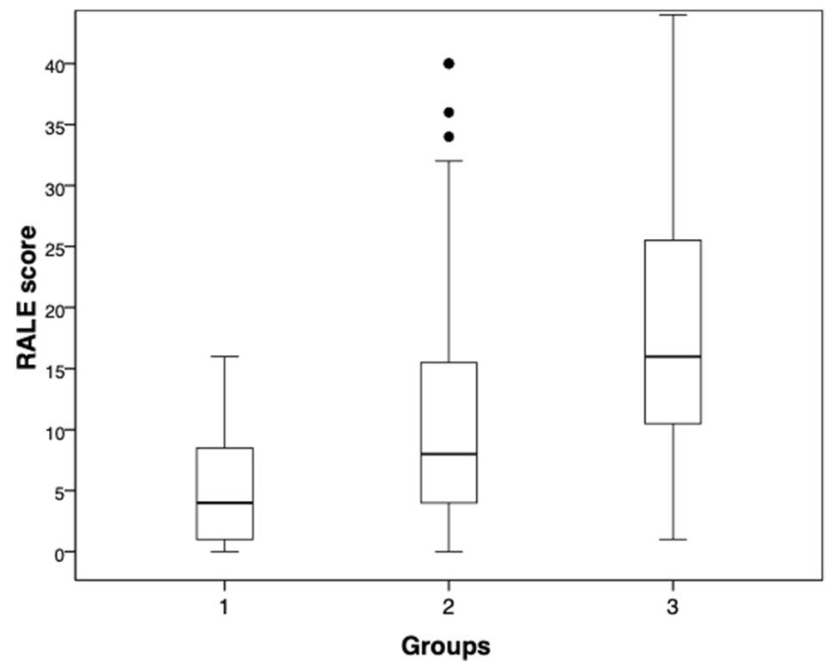

Fig. 2 Box and Whisker plot of RALE score estimated in each group defined by outcome: discharged patients (group 1), hospitalized patients into a medicine department (group 2), hospitalized patients into an intensive care unit (group 3). RALE score showed statistically significant differences between group 1 vs 3 and 2 vs 3 infection. The Italian Society of Radiology (SIRM) recommends using CXR as a first-line imaging tool and reserves to chest $\mathrm{CT}$ others additional roles as the identification of COVID-19 pneumonia typical features in selected cases $[6-8,11]$. Multiple recent studies indicate that CXR may not have the diagnostic power of $\mathrm{CT}$, but it still has a role in managing the pandemic $[6,12,13]$. In fact, although CT has a high sensitivity (around 97-98\%), it has a very low specificity in detecting typical features of SARS-CoV-2 pneumonia $[12,14,15]$. Our study reveals a CXR sensitivity substantially in accordance with the most recent literature $(68.1 \%$ ), where a variability between 69 and $90 \%$ is described $[12,13]$. In our tertiary center, CT examination is usually performed at ED (generally after a CXR) only in specific situations: in case of clinical-radiological discordance (when CXR is negative for infective lung involvement, but there is a high clinical-epidemiological suspect), in case of acute complication (p.e. pulmonary embolism or severe respiratory failure) or after intubation before transporting patient to ICU (Fig. 3). It is operationally much 


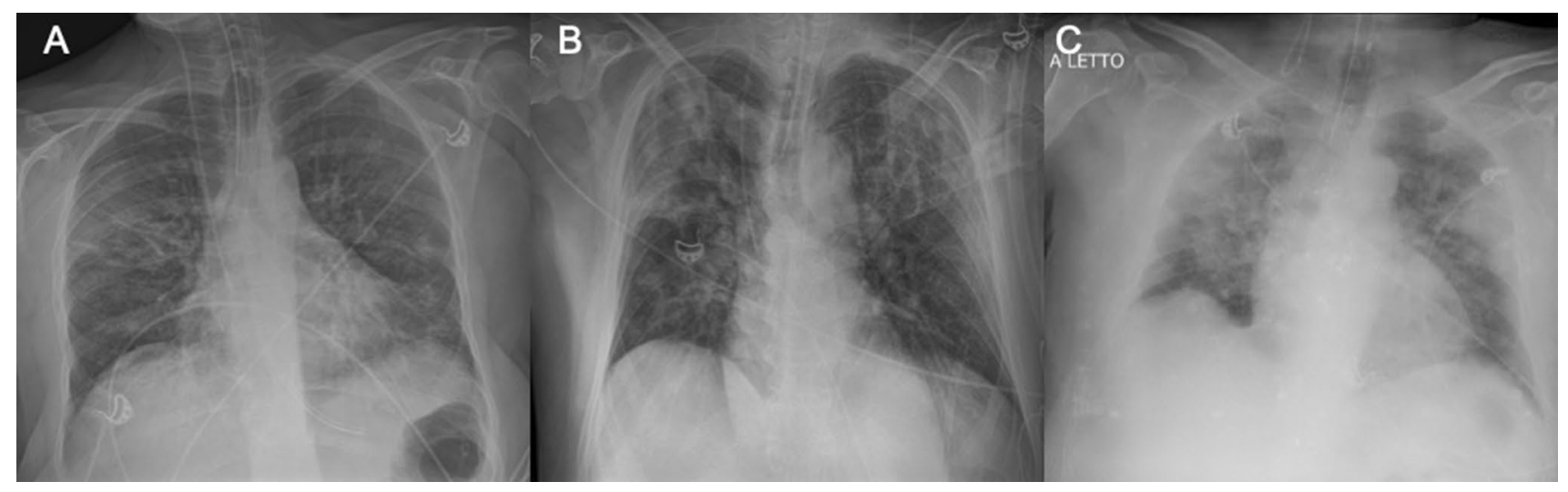

Fig. 3 Chest X-ray in three patients with severe respiratory failure, immediately intubated at the arrival in emergency department and transferred to ICU

more complex to perform CT scans, especially considering the disinfection procedures that have to take place after each examination. For this reason, portable X-ray machine is very useful, inexpensive and radiographs can be taken to the patient's bedside, reducing radiology department's exposure to infection and minimizing the risk of cross-infection.

Our study confirms the main radiological characteristics in COVID-19 patients described in previous studies: In most cases, CXR shows patchy or diffuse reticular-nodular opacities and consolidation, with basal, peripheral and bilateral predominance (Figs. 4, 5). In case of monolateral involvement, right lung is affected more frequently than the left one (58\% vs $42 \%$ ). Our CXR was performed in a period between 2 and 15 days after the onset of symptoms, with cases of more advanced lung involvement in patients around the tenth day of illness. We applied the RALE score, used for the quantification of lung involvement in ARDS [10], in order to standardize and objectively quantify the radiographic report and to produce a prognostic score at the patient's admission. We found a significant statistical correlation between RALE score and patients' outcome, with a RALE score higher than 15 points which correlates with an increased risk of being admitted to ICU. So, the above data confirmed RALE score as a valid standardized prognostic score. In our experience, RALE score has demonstrated to be highly reproducible, as we found with an optimal interobserver reproducibility (ICC: 0.92 [95\% CI 0.88-0.95]).

The role of CXR in clinical monitoring of this patients, especially in ICU, is still debated: Fleischner Society do not recommend daily CXR in stable intubated patients, but it is also true that the disease can progress very quickly $[16,17]$. Our opinion is that radiological imaging should be always associated with clinical and laboratoristic parameters when monitoring the disease course, waiting for readily effective therapies. Obviously, every hospital in the world has its own "radiological" organization and management of the patient with SARS-CoV-2 infection, but it is always necessary to

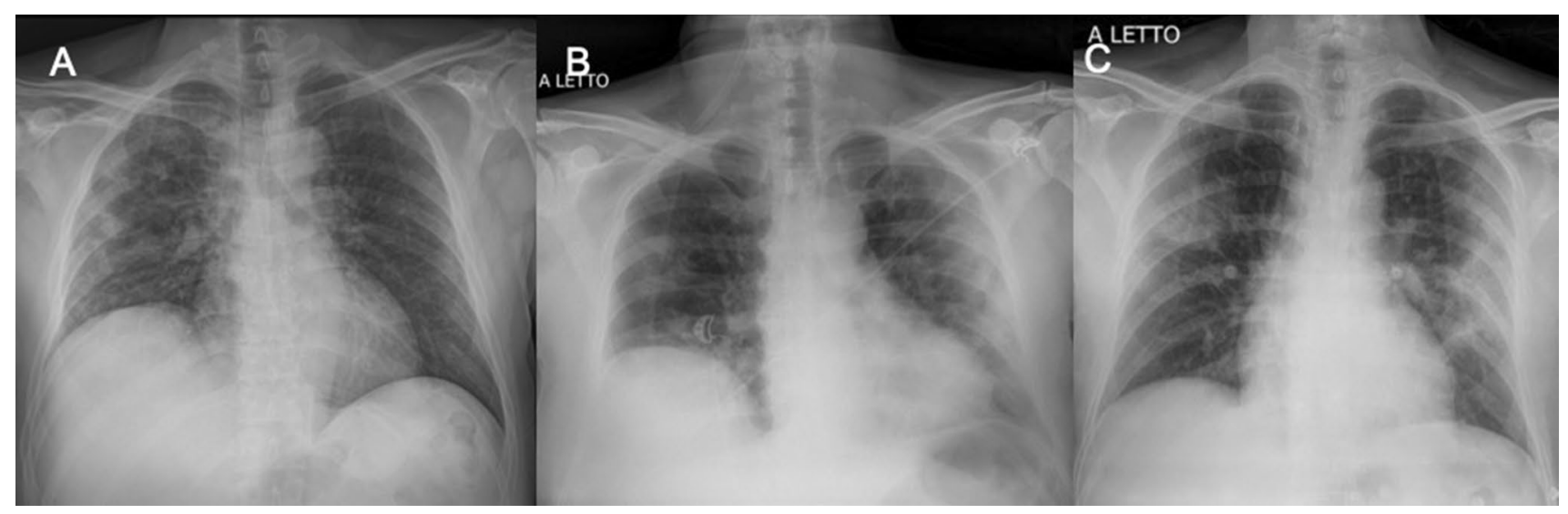

Fig. 4 Peripheral consolidation in COVID-19 pneumonia. Images in A, B and C show three cases of male patients with subpleural consolidations and bilateral involvement 


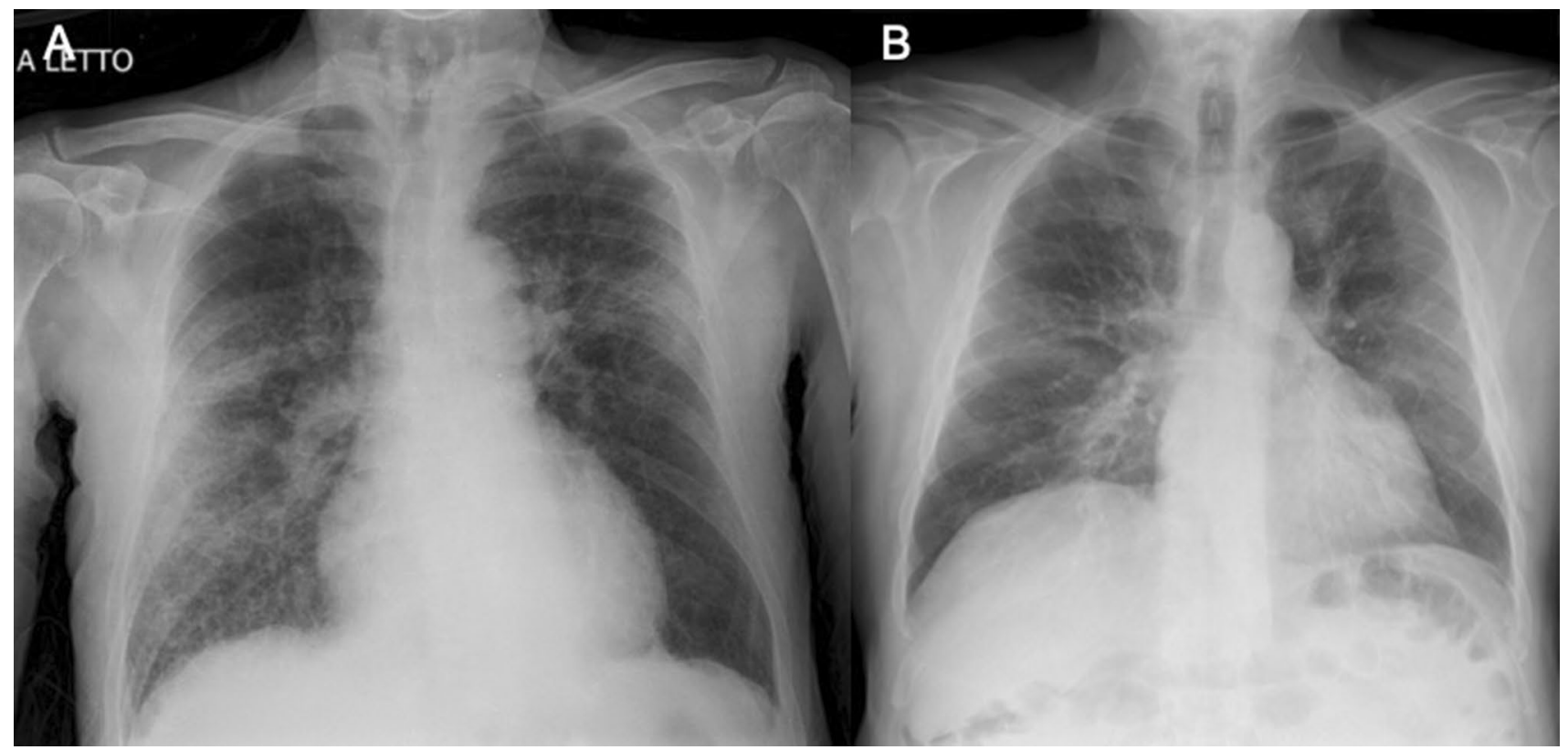

Fig. 5 Interstitial involvement in COVID-19 pneumonia. Two cases of mainly interstitial involvement in Sars-CoV-2 infection. Patient in A shows a prevalent right lung disease with diffuse reticular-nodular

maintain a balance between the safety of health professionals and the diagnostic resources that we can use in this pandemic [16].

Our work has several limitations: first of all, the retrospective nature of the study and the lack of a non-COVID-19 control group in the study of the prognostic score, thus limiting evaluation of sensitivity and specificity of CXR. Moreover, also the lack of comparison between RALE score and patients' comorbidities is a limitation because some clinical data were available only in a small percentage of subjects. In addition, there is a difference in size between the three prognostic groups and a difference in the period of time between the onset of symptoms and CXR's execution. In future, it will be necessary to validate the method considering also these variables and evaluate how the RALE score varies in radiological follow-up. In our study we applied a visual evaluation either in the diagnostic and in the prognostic approach. It would be interesting to continue the study with application of an artificial intelligence (AI) software for a more sophisticated image analysis. Although there are some data about AI and chest CT, neural network applied to chest radiographs needs further investigations and it is too early to apply this new technology in the clinical practice $[7,18,19]$.

In conclusion, we describe the main features of COVID19 thoracic involvement on CXR in our cohort of patients. In our experience, baseline CXR had a sensitivity of $67.1 \%$. The RALE score can be used in the emergency setting as a quantitative method of the extent of SARS-CoV-2 thickening of peribroncho-vascular interstitium. Patient in B shows a diffuse ground glass opacity mainly in the perihilar and subpleural region bilaterally

pneumonia, correlating with an increased risk of ICU admission. The results of our study could help radiologists in identifying the highest risk patients, allowing for timely initiation of treatments currently available against SARSCoV-2 infection.

\section{Compliance with ethical standards}

Conflict of interest The authors declare that they have no conflict of interest related to the publication of this article.

Ethical approval All procedures performed in studies involving human participants were in accordance with the ethical standards of the institutional and/or national research committee and with the 1964 Helsinki Declaration and its later amendments or comparable ethical standards.

Informed consent Informed consent was obtained from all individual participants included in the study. The study protocol was submitted to the ethics committee of our referring center.

\section{References}

1. Zhu N, Zhang D, Wang W et al (2020) A novel coronavirus from patients with pneumonia in China, 2019. N Engl J Med. https:// doi.org/10.1056/NEJMoa2001017

2. Wang C, Horby PW, Hayden FG, Gao GF (2020) A novel coronavirus outbreak of global health concern. Lancet 395(10223):470-473 
3. Chen N, Zhou M, Dong X et al (2020) Epidemiological and clinical characteristics of 99 cases of 2019 novel coronavirus pneumonia in Wuhan, China: a descriptive study. Lancet 395(10223):507-513

4. Yang W, Sirajuddin A, Zhang X et al (2020) The role of imaging in 2019 novel coronavirus pneumonia (COVID-19). Eur Radiol. https://doi.org/10.1007/s00330-020-06827-4

5. Zhou A, Wang Y, Zhu T, Xia L (2020) CT features of Coronavirus disease 2019 (COVID-19) pneumonia in 62 patients in Wuhan, China. Am J Roentgenol 214:1-8

6. Giovagnoni A (2020) Facing the COVID-19 emergency: we can and we do. Radiol Med 125(4):337-338

7. Neri E, Miele V, Coppola F, Grassi R (2020) Use of CT and artificial intelligence in suspected or COVID-19 positive patients Statement of the Italian Society of Medical and Interventional Radiology. Radiol Med. https://doi.org/10.1007/s11547-020-01197-9

8. ACR recommendations for the use of chest radiography and computed tomography (CT) for suspected COVID-19 infection. American College of Radiology. https://www.acr.org/Advocacyand-Economics/ACR-Position-Statements/Recommendations-forChest-Radiography-and-CT-for-Suspected-COVID-19-infection. Updated March 222020.

9. Hansell DM, Bankier AA, MacMahon H et al (2008) Fleischner Society: glossary of terms for thoracic imaging. Radiology 246(3):697-722. https://doi.org/10.1148/radiol.2462070712

10. Warren MA, Zhao Z, Koyama T et al (2018) Severity scoring of lung edema on the chest radiograph is associated with clinical outcomes in ARDS. Thorax. https://doi.org/10.1136/thoraxjnl2017-211280

11. https://www.sirm.org/wp-content/uploads/2020/03/DI-COVID -19-documento-intersocietario.pdf. Accessed 23 Apr 2020 (documentinItalian)

12. Choi H, Qi X, Yoon SH et al (2020) Extension of coronavirus disease 2019 (CVID-19) on chest CT and implications for chest radiograph interpretation. Radiology. https://doi.org/10.1148/ ryct.2020200107

13. Wong HYF, Lam HYS, Fong AHT et al (2020) Frequency and distribution of chest radiographic findings in COVID-19 positive patients. Radiology. https://doi.org/10.1148/radiol.2020201160

14. Ai T, Yang Z, Hou HY et al (2020) Correlation of chest CT and RT-PCR testing in coronavirus disease 2019 (COVID-19) in China: a repost of 1014 cases. Radiology. https://doi.org/10.1148/ radiol.2020200642

15. Fang Y, Zhang HQ, Xie J et al (2020) Sensitivity of chest CT for COVID-19: comparison to RT-PCR. Radiology. https://doi. org/10.1148/radiol.2020200432

16. Rubin GD, Ryerson CJ, Haramati LB et al (2020) The role of chest imaging in patient management during the COVID-19 pandemic: a multinational consensus statement from the Fleischner Society. Radiology. https://doi.org/10.1148/radiol.2020201365

17. Fichera G, Stramare R, de Conti G et al (2020) It's not over until it's over: the chameleonic behavior of COVID-19 over a six-day period. Radiol Med. https://doi.org/10.1007/s11547-020-01203-0

18. Li L, Qin L, Xu Z et al (2020) Artificial intelligence distinguishes COVID-19 from community acquired pneumonia on chest CT. Radiology. https://doi.org/10.1148/radiol.2020200905

19. Santosh KC (2020) AI-driven tools for coronavirus outbreak: need of active learning and cross-population train/test models on multitudinal/multimodal data. J Med Syst 44(5):93

Publisher's Note Springer Nature remains neutral with regard to jurisdictional claims in published maps and institutional affiliations. 